Open Access

\title{
Performance analysis of gain ratio power allocation strategies for non-orthogonal multiple access in indoor visible light communication networks
}

\author{
Siyu Tao* (D), Hongyi Yu, Qing Li and Yanqun Tang
}

\begin{abstract}
Non-orthogonal multiple access (NOMA) is a promising method for enhancing the throughput in visible light communication (VLC) networks. In NOMA, signal power domain control, called gain ratio power allocation (GRPA), can significantly improve the user sum rate with full-time frequency resource utilization. In indoor NOMA-VLC networks, the scenario in which users are covered by VLC illuminants on the ceiling is typical. First, this paper proposes a novel GRPA strategy for a single VLC cell. Second, due to the difficulty of direct comparisons between our and previously reported GRPA strategies, this paper presents an alternative lower bound for comparability. Third, in two- and threeuser cases, this paper analytically demonstrates that our NOMA-VLC GRPA strategy outperforms the aforementioned strategy, when successive interference cancellation (SIC) is used. Moreover, in the multi-user case, experimental results show that the average user data rate (AUDR) of multi-user cases under our strategy is better than that under the previous strategy. Finally, our paper provides both analytic and numerical results to prove that VLC single-cell system throughput under our strategy is better than that under the previous strategy. The proposed alternative lower bound is also proved to fit the original NOMA-VLC GRPA target asymptotically based on indoor VLC channels. Furthermore, to achieve the same AUDR, our strategy supports worse received conditions than does the previous strategy.
\end{abstract}

Keywords: Visible light communication networks, Non-orthogonal multiple access, Gain ratio power allocation, Alternative lower bound, Single cell

\section{Introduction}

While current wireless local area network (WLAN) implementations reach the wireless fidelity (WiFi) consumer market with triple-band demand, optical wireless communications (OWC), specifically those based on indoor visible light communication (VLC) technology or light fidelity (LiFi), possess a broad spectrum and ubiquitous layout. When used for WLAN, VLC [1] utilizes a lightemitting diode (LED) to transmit visible light data. From the physical layer to the network layer, studies on VLC have gradually explored networking issues related to the design and maintenance of VLC networks. VLC links have directionality and vulnerability characteristics, owing to

*Correspondence: peachforworking@foxmail.com

National Digital Switching System Engineering and Technological Research Center (NDSC), 450001 Zhengzhou, People's Republic of China the straight propagation and common obstruction of visible light, respectively. Therefore, research on VLC networking encounters several challenges related to these characteristics that differ from those of either wireless fidelity or femtocell networks, such as illuminant layout design, VLC multi-user access, visible light channel resource allocation, and mobility management.

Next-generation wireless communication involves three types of density requirements [2], i.e., high user density, high traffic density and high access point (AP) deployment. Regarding high user density, scenarios such as those involving several users at home, dozens of users in the office or hundreds of users in the mall may evolve into an indoor ultra dense network (UDN) if user density increases. For the example of an office, the corresponding employee density is 0.25 person $/ \mathrm{m}^{2}$. With respect to high traffic density, sharing high-definition videos 
based on indoor virtual reality will lead to high traffic density reaching nearly $10 \mathrm{Mbps} / \mathrm{m}^{2}$. Regarding high AP density, in traditional cellular networks, the cellular range is greater than $500 \mathrm{~m}$ and fewer than approximately 3 to 5 base stations cover $1 \mathrm{~km}^{2}$, which cannot ensure sufficiently high throughput for crowded and building-blocked users. For traditional macrocells and femtocells [3, 4], the continuous deployment of radio services, such as base station and communication standard uses, cause the limited bandwidth usage and rare resource access.

Correspondingly, indoor VLC APs with UDN networking is an efficient way to improve the system traffic capacity and user experience [1], particularly in hotspot areas [2]. For high user density, the indoor VLC network is an important small cell scheme for increasing the coverage of remote public devices and maintaining high-speed communication in the last-meter prolongation of backbone networks. Ubiquitous VLC illuminants have certain demands for dividing indoor crowds into several independent subclusters or single cells [5]. For high traffic density, VLC considers broad optical spectrum resources to make wireless broadband local area communication possible and meet very high area traffic requirements $[1,6]$. For high AP density, the AP deployment of VLC systems in a room can be dense and arbitrary [7], which increases the flexibility of deployments [2]. However, VLC remains challenging in several respects. Because VLC involves worse path loss and higher frequency than $\mathrm{mm}$ wave communications do, the sizes of VLC cells may be reduced, leading to the concept of VLC attocells $[1,8]$ that are smaller than domestic microcells or femtocells. In the interior of a room, VLC cells, as an ultra dense local network, may lead to ultra dense interference of inter and intra VLC cells with a smaller coverage than radio frequency (RF) communication. Hence, for capacity improvement and interference mitigation, novel multiple user access and power control mechanisms should be taken in account within VLC attocells.

To maximize the advantages of high-density coverage within a VLC cell, non-orthogonal multiple access (NOMA) [9-12] as a promising candidate for nextgeneration wireless networks, has been introduced into VLC networks for promoting of network system throughput. Compared with orthogonal multiple access (OMA) $[5,13]$, NOMA can exploit the entire bandwidth for the time-frequency utilization of all degrees of freedom to increase spectral efficiency. Furthermore, NOMA is designed to allocate more signal power to users experiencing poor channel conditions. It is intended to enhance the data rate of edge users and alleviate the near-far effect without degrading the performance of central users. In NOMA, gain ratio power allocation (GRPA) [5, 11, 14] considers user channel conditions to ensure efficient and fair power allocation of LED transmitted signal power, while enhancing the achievable throughput in VLC networks. In RF and VLC, the power allocation of NOMA extends research goals in several major respects, such as user fairness $[15,16]$, channel allocation $[17,18]$, user scheduling $[7,9,18]$, energy efficiency $[9,19-21]$, quality of service (QoS) requirement $[9,10,22,23]$, and sum rate maximization $[11,16,24,25]$.

GRPA studies related to both NOMA and VLC are listed as follows. In [9], a power allocation strategy considering residual interference was optimized by the QoS constraint and numerical search with both max-min and max-sum rate criteria. In [10], analytical expressions of user outage probability for guaranteed QoS and an ergodic sum rate for opportunistic best-effort service were derived. In $[11,26]$, a GRPA strategy based on a power law criterion that considers user channel conditions was presented. In [15], researchers investigate a downlink power control strategy involving user fairness and unique intensity constraints for optical modulations. In [23], comprehensive numerical results verify the superiority of NOMA-VLC in achieving a satisfactory QoS target rate by random receiver orientation. Vertical angle information can compensate for the loss in user rates by incorporating suitable power allocation coefficients, which are inversely proportional to the channel quality of the respective NOMA user.

In summary, solutions are of the following two types, i.e., numerical search and strategy design. It is difficult to yield the optimal solution with either method, because the power allocation optimization problem for NOMA is mathematically NP-hard [17, 18, 27]. This paper focuses on the development of a centralized power control strategy within a VLC single cell. Although the implementation of a centralized controller in dense multi-tier heterogeneous wireless networks is not feasible [4], the centralized control may expediently handle with the channel status information of connected users [28] and thereby reduce interference for careful resource scheduling in small cells. Even in a single cell, very high user density can introduce multi-user interference, which deserves further investigation as well. Therefore, to better support advanced resource management, the centralized management of VLC APs is necessary to measure users' channel environment and implement signal power allocation.

However, GRPA problems in NOMA-VLC [29] networks have not yet been solved. In view of the difficulty of obtaining a generalized GRPA solution, the method of numerical search has the shortcoming of expensive computing, but using such strategies is convenient for real-time processing. Therefore, in this paper, we focus on GRPA strategies of NOMA in a VLC network and achieve the following works. 
- Due to the complex mathematical expansion of a NOMA-VLC throughput target, this paper provides an alternative lower bound as an approximate target to evaluate the performance of the VLC user data rate for NOMA mathematical convenience.

- To demonstrate the accuracy of lower bound substitution in NOMA-VLC networks, details such as asymptotic properties and performance boundary are analyzed. We discover that the substitutability of our alternative bound is feasible for VLC channels.

- This paper presents a GRPA strategy based on VLC channel gains. Considering the indoor VLC network environment, the home is a typical application scenario, which involves a limited room size and few users. For two- and three-user cases, we derive an analytic proof that shows that our GRPA strategy is better than the reported strategy in previous papers $[11,26]$.

- For a great number of users, a performance analysis between the previously reported strategy and our strategy is performed by numerical simulations. Experimental results show that the average user data rate of NOMA is greater than that of OMA, and our GRPA strategy outperforms that previous strategy with the number of users not limited to three.

\section{Methodology}

\subsection{Channel gain of VLC}

The entire coverage of a single AP LED suspended from the ceiling of a room is defined as a LiFi single cell. The users within a cell use the photo diode (PD) as the VLC signal receiver. Due to the low mobility and line of sight (LOS) within a limited indoor area, the Doppler frequency shift and multi-path effect can be disregarded. Hence, the indoor VLC signal model can be referred to as a Lambertian radiation model [6]. Considering that the VLC signal in the LOS path is the main energy component, we denote the VLC channel gain for the $k$-th user as (1) shows.

$$
h_{k}=\frac{(m+1) A}{2 \pi d_{k}^{2}} \cdot \cos ^{m}\left(\phi_{k}\right) \cdot T_{f i l t e r} \cdot g\left(\psi_{k}\right) \cdot \cos \left(\psi_{k}\right)
$$

where $m$ is the order of Lambertian emission, $A$ is the physical area of the PD detector, $d_{k}$ is the distance between an LED transmitter and a PD receiver of the $k$ th user, $\phi_{k}$ is the angle of irradiance of the $k$-th user, $\psi_{k}$ is the angle of incidence of the $k$-th user, $T_{\text {filter }}$ is a constant of an optical filter gain, and $g\left(\psi_{k}\right)$ is the gain of an optical concentrator of the $k$-th user.

In addition, $m$ and $g\left(\psi_{k}\right)$ are given by (2) and (3), respectively. $\Phi_{1 / 2}$ is the semi-angle of the LED, $\Psi_{F O V}$ is the width of the angle field of vision at a PD receiver, and $n$ is a constant of a refractive index.

$$
\begin{aligned}
m & =-\frac{1}{\log _{2}\left(\cos \left(\Phi_{1 / 2}\right)\right)} \\
g\left(\psi_{k}\right) & = \begin{cases}\frac{n^{2}}{\sin ^{2}\left(\Psi_{F O V}\right)} & , 0 \leq \psi_{k} \leq \Psi_{F O V} \\
0 & , \psi_{k}>\Psi_{F O V}\end{cases}
\end{aligned}
$$

As shown in Fig. 1, the height $L$ and the radius $r_{k}$ of the $k$-th user can be substituted for $d_{k}$ in (4), where $\rho$ is a constant equal to $\frac{A \cdot T_{\text {filter }} \cdot g\left(\psi_{k}\right)}{2 \pi}$.

$h_{k}=\frac{\left(\frac{A \cdot T_{\text {filter }} \cdot g\left(\psi_{k}\right)}{2 \pi}\right) \cdot(m+1) \cdot L^{m+1}}{\left(r_{k}^{2}+L^{2}\right)^{\frac{m+3}{2}}}=\frac{\rho \cdot(m+1) \cdot L^{m+1}}{\left(r_{k}^{2}+L^{2}\right)^{\frac{m+3}{2}}}$

For the PD receiver of the $k$-th user, the received electrical signal power $P R_{k}$ is derived by the transmitted optical signal power $P T_{k}$ and optical-electrical conversion efficiency constant $\gamma$ as follows.

$$
P R_{k}=\gamma \cdot h_{k} \cdot P T_{k}
$$

\subsection{GRPA of NOMA in VLC}

In NOMA, users with poor channel quality are allocated more signal power than others to guarantee the data rate of cell-edge users. If the number of users within a VLC cell is $K$, the channel gains of the users can be listed in increasing order as follows.

$$
h_{1}<h_{2}<\cdots<h_{k}<\cdots<h_{K}
$$

Based on the principle of NOMA, the users in a cell share the fixed transmitted optical signal power $P T$ and are reassigned the respective signal power as follows. The parameter $a_{k}$ is the factor of the redistributive signal power.

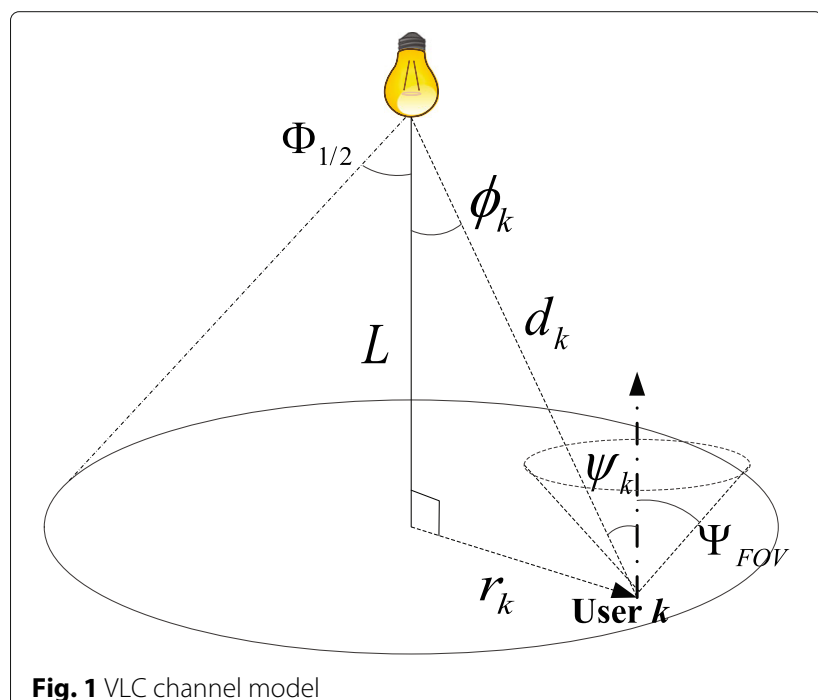


$a_{1} \cdot P T>a_{2} \cdot P T>\cdots>a_{k} \cdot P T>\cdots>a_{K} \cdot P T$, s.t. $\sum_{k=1}^{K} a_{k}=1$

The received signal transmitted from the LED to the $k$-th user is assumed in (8), where $s_{k}$ denotes the onoff keying (OOK) signal and $n_{k}$ denotes the zero-mean Gaussian noise caused by many independent sources $[7,10,25,30,31]$.

$$
Y_{k}=\gamma \cdot P T \cdot h_{k} \cdot \sum_{i=1}^{K} a_{i} \cdot s_{i}+n_{k}
$$

In VLC systems, intensity modulation and direct detection (IM/DD) is employed for signal modulation and detection. It is reasonable to model an IM/DD channel as an additive white Gaussian noise channel [7, 31].

\subsection{User data rate bound}

The user data rate can be expressed diversely under different constraints as follows.

- Several studies $[9,10,15]$ have continued to use the Shannon capacity formula, which may not be accessible without the maximizing input distribution. However, the bound can be referred to as the achievable bound. The signal to interference plus noise ratio (SINR) for the $k$-th user in (9) is usually used to measure the VLC user data rate $[9,23,32,33]$, where $N_{0}$ is a constant noise power spectral density and $B$ is a constant VLC bandwidth.

$$
\operatorname{SINR}_{k}=\frac{\left(\gamma \cdot h_{k} \cdot a_{k} \cdot P T\right)^{2}}{\sum_{i=k+1}^{K}\left(\gamma \cdot h_{k} \cdot a_{i} \cdot P T\right)^{2}+N_{0} B}, k<K
$$

- On the other hand, no exact closed-form expression for the VLC channel capacity has yet been established due to constraints of the input VLC signals. Instead, the $\mathrm{u}$ se of several tight capacity bounds [7, 25, 30, 31, 34] is more practical to approach the channel capacity in VLC performance analysis. Due to the different constraints imposed on the VLC system, the expression of the lower bound is different.

If input VLC signals with an average power constraint are assumed, different data rate bounds in the two cases of inter-symbol interference (ISI) [7, 16, 30, 34] and ISI-free $[31,34]$ are deduced. If Successive Interference Cancellation (SIC) $[10,11,18,29]$ is deployed in VLC receivers, the NOMA-VLC typical scenario is a single cell with interuser interference. Hence, according to the conditions of ISI, the achievable data rate of NOMA-VLC users for the $k$-th user can be approximately indicated as (10), where the Euler number $e$ and $\pi$ are the determined values.
Note that the transmit symbols of the baseband signals are uniformly distributed over the symbol interval $[-1,1]$.

$$
\begin{gathered}
\text { Rate }_{k}\left(h_{k}, a_{k}\right)=\frac{B}{2} \cdot \log _{2}\left(1+\frac{2}{\pi e} \cdot \operatorname{SINR}_{k}\right), k<K \\
\underset{a_{1}, \cdots, a_{k}, \cdots, a_{K}}{\arg \max } \sum_{k=1}^{K} \text { Rate }_{k}\left(h_{k}, a_{k}\right)
\end{gathered}
$$

Based on the VLC channel gain $h_{k}$, the optimization target of GRPA is to select the optimal parameter $a_{k}$ for the throughput maximization of the VLC network system as follows.

$$
\text { Rate }_{k}{ }_{k}\left(h_{k}, a_{k}\right)=B^{\prime} \cdot \log _{2}\left(1+\eta \cdot \operatorname{SINR}_{k}\right), k<K
$$

For the convenience in establishing the general mathematical forms of VLC relationships, this paper proposes (12) of Shannon theory $[9,10,15]$ to perform a comparison analysis between NOMA-VLC GRPA strategies, which yields results equivalent to those produced by strategy comparisons. The reasons are as follows.

- NOMA utilizes the whole available bandwidth, whose efficiency can be normalized. We can regard the bandwidth as a common factor that can be extracted and treat $\frac{2}{\pi e}$ as a constant $\eta$.

- For the same bound, two strategies denoted as OurS and PLS would generate two numerical SINR values denoted as $\Lambda_{1}$ and $\Lambda_{2}$, respectively. For the lower bounds (10) and (12), the comparison relationships can be indicated as (13) and (14).

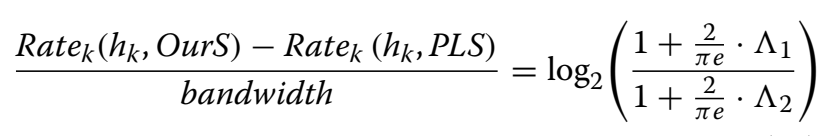

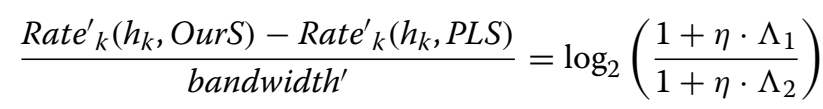

It is clear that the contrasting relationships of positives and negatives discussed for the bound (10) is equivalent to those discussed for the bound (12). Although (10) and (12) have different physical meanings, the consistency of those mathematical forms to a certain extent reflects that both have common laws in the derivation of mathematical relationships. For the abovementioned reasons, we can use Shannon theory for convenience in establishing equivalence mathematically and ensure that our derivation applies to both (10) and (12).

\subsection{Motivation of GRPA strategy design}

There are two types of methods for solving the abovementioned problem: the numerical search method in 
which the mathematical form of the optimization target hardly yields an analytical solution and strategy design, which is adopted in this paper. To focus on the design of GRPA strategies in NOMA-VLC networks [11, 26], presented ideas in which the design of the power allocation factor may follow the channel gains. Several studies [11, 23, 26, 35, 36] on GRPA strategy design are principally based on the inverse ratio, with an ascending order distribution of the channel gain, as similar as a power law strategy.

- Power law strategy (PLS) [11, 26]: Previous studies' factor strategy is discussed in (15).

$$
a_{k}=\left\{\begin{array}{l}
\frac{1}{1+\left(\frac{h_{1}}{h_{2}}\right)^{2}+\left(\frac{h_{1}}{h_{2}}\right)^{2} \cdot\left(\frac{h_{1}}{h_{3}}\right)^{3} \cdots+\prod_{i=2}^{K}\left(\frac{h_{1}}{h_{i}}\right)^{i}}, k=1 \\
\frac{\prod_{i=2}^{K}\left(\frac{h_{1}}{h_{i}}\right)^{i}}{1+\left(\frac{h_{1}}{h_{2}}\right)^{2}+\left(\frac{h_{1}}{h_{2}}\right)^{2} \cdot\left(\frac{h_{1}}{h_{3}}\right)^{3} \cdots+\prod_{i=2}^{K}\left(\frac{h_{1}}{h_{i}}\right)^{i}}, 2 \leq k \leq K
\end{array}\right.
$$

- Our strategy (OurS): This paper's GRPA factor strategy is discussed in (16). Using $h_{k}$ to compute $a_{k}$, this paper presents a revision for degrading the power counting of $\frac{h_{1}}{h_{k}}$ as our strategy.

$$
a_{k}=\left\{\begin{array}{l}
\frac{1}{1+\left(\frac{h_{1}}{h_{2}}\right)+\cdots+\left(\frac{h_{1}}{h_{k}}\right)}, k=1 \\
\frac{\left(\frac{h_{1}}{h_{k}}\right)}{1+\left(\frac{h_{1}}{h_{2}}\right)+\cdots+\left(\frac{h_{1}}{h_{k}}\right)}, 2 \leq k \leq K
\end{array}\right.
$$

In the following sections, this paper provides rigorous proofs of two- and three- user cases to demonstrate that our strategy is better than the power law strategy and discusses the result of numerical simulations to demonstrate that our method retains its inherent advantages with more than three users.

\section{Performance evaluation of GRPA strategy 3.1 NOMA-VLC GRPA in the two-user case \\ 3.1.1 Two-user basic throughput model}

Without loss of generality, this subsection focuses on the case of two users. The VLC channel gains of two users can be sorted as $0<h_{1}<h_{2}$ with the normalization of the fixed transmitted optical signal power $P T$. For the equivalent and general mathematical model, (12) without loss of generality $(\eta=1)$ is selected to transform the problem into (17), where the constraint follows $a_{1}+a_{2}=1,1>$ $a_{1}>a_{2}>0$.

$$
\begin{aligned}
& \underset{a_{1}, a_{2}}{\arg \max }\left[\log _{2}\left(1+\frac{\left(\gamma h_{1} a_{1}\right)^{2}}{\left(\gamma h_{1} a_{2}\right)^{2}+N_{0} B}\right)\right. \\
& \left.+\log _{2}\left(1+\frac{\left(\gamma h_{2} a_{2}\right)^{2}}{N_{0} B}\right)\right]
\end{aligned}
$$

The two users' problem of (17) is equivalent to $\arg \max S_{2}$ including (18).

$$
S_{2}=\left(1+\frac{\left(\gamma h_{1} a_{1}\right)^{2}}{\left(\gamma h_{1} a_{2}\right)^{2}+N_{0} B}\right) \cdot\left(1+\frac{\left(\gamma h_{2} a_{2}\right)^{2}}{N_{0} B}\right)
$$

\subsubsection{Our alternative bound in the two-user model}

The mathematical form of (18) creates a certain difficulty in comparing different strategies. This paper presents the asymptotic lower bound of (18) to ensure the strategies are comparable as shown in (19). Note that the hypothesis that (19) is the asymptotic lower bound of (18) is supported by the relationship among VLC channel gains.

$$
S_{2}^{\prime}=1+\frac{\left(\gamma h_{1} a_{1}\right)^{2}+\left(\gamma h_{2} a_{2}\right)^{2}}{N_{0} B}
$$

First, the accuracy of the lower bound can be certified by (20).

$$
S_{2}-S_{2}^{\prime}=\frac{\left(\gamma^{2} h_{1} a_{1} a_{2}\right)^{2} \cdot\left(h_{2}^{2}-h_{1}^{2}\right)}{N_{0} B \cdot\left[\left(h_{1} a_{2}\right)^{2}+N_{0} B\right]}>0
$$

Second, (21) can be obtained by (4), where $\Omega$ is indicated by (22). Equations (21) and (22) ensure that the asymptotic behaviour of an asymptotic lower bound should be as homologous as an approximate objective, which guarantees that the analysis of each strategy can be performed rationally and comparably. Once VLC APs are deployed, the light angles and layout are determined. Therefore, according to the relationship $h_{1}<h_{2}, \Omega_{1}>\Omega_{2}$ can be given as well as $r_{1}>r_{2}$.

$$
\begin{aligned}
S_{2}-S_{2}^{\prime} & =\frac{\left(h_{1} a_{2}\right) \cdot\left(\gamma^{2} a_{1}\right)^{2} \cdot\left(h_{2}^{2}-h_{1}^{2}\right)}{N_{0} B \cdot\left[h_{1} a_{2}+\frac{N_{0} B}{h_{1} a_{2}}\right]} \\
& \leq \frac{\left(h_{1} a_{2}\right) \cdot\left(\gamma^{2} a_{1}\right)^{2} \cdot\left(h_{2}^{2}-h_{1}^{2}\right)}{\left(N_{0} B\right)^{\frac{3}{2}}} \\
& =\frac{\left(\gamma^{2} a_{1}\right)^{2} a_{2}\left(\rho(m+1) L^{m+1}\right)^{3}}{\left(N_{0} B\right)^{\frac{3}{2}}} \cdot\left(\frac{\Omega_{1}^{2}-\Omega_{2}^{2}}{\Omega_{1}^{3} \cdot \Omega_{2}^{2}}\right)
\end{aligned}
$$

$$
\Omega_{1}=\left(r_{1}^{2}+L^{2}\right)^{\frac{m+3}{2}}, \Omega_{2}=\left(r_{2}^{2}+L^{2}\right)^{\frac{m+3}{2}}
$$

Third, the gap between $S_{2}$ and $S_{2}^{\prime}$ is analyzed.

- When the number of user $K$ increases, the limited cell coverage becomes crowded. If we choose any two users from the total $K$ users, two users' radii may be approximate and two channel gains may be converging; thus, (23) can be inferred.

$$
\lim _{K \rightarrow+\infty,\left(r_{1}-r_{2}\right) \rightarrow 0}\left(S_{2}-S_{2}^{\prime}\right)=0
$$

- For the case in which the number of users $K$ is two, we analyze the performance difference between two 
targets in the worst communication situation to demonstrate the compact boundary of our target in (24).

$$
\begin{aligned}
& \lim _{K=2, r_{1}>r_{2}}\left(S_{2}-S^{\prime}{ }_{2}\right) \\
& \leq \lim _{K=2, r_{1}>r_{2}} \frac{\left(\gamma^{2} a_{1}\right)^{2} a_{2}\left(\rho(m+1) L^{m+1}\right)^{3}}{\left(N_{0} B\right)^{\frac{3}{2}}} \cdot\left(\frac{1}{\Omega_{1} \cdot \Omega_{2}^{2}}-\frac{1}{\Omega_{1}^{3}}\right) \\
& \leq\left[1-\left(\frac{r_{2}^{2}+L^{2}}{r_{1}^{2}+L^{2}}\right)^{m+3}\right] \cdot S_{2}
\end{aligned}
$$

- Based on the definition of an asymptotic lower bound and the pre-condition of (23), (25) can be proven to indicate the uniform asymptotic relationship between $S_{2}$ and $S_{2}^{\prime}$.

$$
\begin{aligned}
& \lim _{K \rightarrow+\infty, h_{1} \rightarrow h_{2}} \frac{S_{2}}{S_{2}^{\prime}} \\
& =\lim _{K \rightarrow+\infty}\left(\frac{\left(\gamma h_{1} a_{1}\right)^{2}+\left(\gamma h_{1} a_{2}\right)^{2}+N_{0} B}{\left(\gamma h_{1} a_{1}\right)^{2}+\left(\gamma h_{2} a_{2}\right)^{2}+N_{0} B} \cdot \frac{\left(\gamma h_{2} a_{2}\right)^{2}+N_{0} B}{\left(\gamma h_{1} a_{2}\right)^{2}+N_{0} B}\right)=1
\end{aligned}
$$

In summary, (19), as a performance bound of the accessible user rate, can be substituted for (18) while retaining the same functional behaviour.

\subsubsection{Strategy comparison of two-user model}

For the target $S_{2}^{\prime}$, the comparison is equivalent to (26), where $a_{1}+a_{2}=1$.

$$
\underset{a_{1}, a_{2}}{\arg \max } S_{2}^{\prime} \Leftrightarrow \underset{a_{1}, a_{2}}{\arg \max } S_{2}^{\prime \prime}{ }_{2}, S^{\prime \prime}{ }_{2}=\left(h_{1} a_{1}\right)^{2}+\left(h_{2} a_{2}\right)^{2}
$$

When the number of users is two, the factor allocation of the power law strategy (PLS) is indicated as (27), and the system throughput can be indicated by (28).

$$
\begin{gathered}
P L S: a_{1}=\frac{h_{2}^{2}}{h_{1}^{2}+h_{2}^{2}}, a_{2}=\frac{h_{1}^{2}}{h_{1}^{2}+h_{2}^{2}} \\
P L \_S_{2}^{\prime \prime} \triangleq S_{2}^{\prime \prime}\left(P L S: a_{1}, a_{2}\right)=\frac{h_{1}^{2} h_{2}^{2}}{\left(h_{1}^{2}+h_{2}^{2}\right)}
\end{gathered}
$$

Our strategy is expressed as (29) and (30).

OurS : $\quad a_{1}=\frac{h_{2}}{h_{1}+h_{2}}, a_{2}=\frac{h_{1}}{h_{1}+h_{2}}$

Our_S $S_{2}^{\prime \prime} \triangleq S_{2}^{\prime \prime}\left(\right.$ OurS $\left.: a_{1}, a_{2}\right)=\frac{2 h_{1}^{2} h_{2}^{2}}{\left(h_{1}+h_{2}\right)^{2}}=\frac{h_{1}^{2} h_{2}^{2}}{\frac{1}{2}\left(h_{1}+h_{2}\right)^{2}}$

Based on mean value inequality, (31) is proved. Therefore, for the two-user sum rate in a VLC network system, our strategy is better than the power law strategy.

$$
\begin{aligned}
& \frac{1}{2}\left(h_{1}+h_{2}\right)^{2}<\left(h_{1}^{2}+h_{2}^{2}\right) \Leftrightarrow 0<\left(h_{1}-h_{2}\right)^{2} \\
& \Rightarrow P L \_S_{2}^{\prime \prime}<O u r \_S_{2}^{\prime \prime}
\end{aligned}
$$

\subsection{NOMA-VLC GRPA in the three-user case \\ 3.2.1 Three-user GRPA model}

For a three-user model, the alternative bound target can be improved as indicated by (32) and (33), where $h_{1}<$ $h_{2}<h_{3}$ and $a_{1}>a_{2}>a_{3}$ are pre-conditions. As a reasonable approximation, (33) can be verified by (23)-(25) as well.

$$
S_{3}^{\prime}=1+\frac{\left(\gamma h_{1} a_{1}\right)^{2}+\left(\gamma h_{2} a_{2}\right)^{2}+\left(\gamma h_{3} a_{3}\right)^{2}}{N_{0} B}
$$

$\underset{a_{1}, a_{2}, a_{3}}{\arg \max } S_{3}^{\prime} \Leftrightarrow \underset{a_{1}, a_{2}, a_{3}}{\arg \max } S^{\prime \prime}{ }_{3}, S^{\prime \prime}{ }_{3}=\left(h_{1} a_{1}\right)^{2}+\left(h_{2} a_{2}\right)^{2}+\left(h_{3} a_{3}\right)^{2}$

The factor allocation forms in the three-user model are as follows.

- Power law strategy: The three-user factor forms are given by (34), and the target is indicated by (35).

$$
\begin{aligned}
& a_{1}=\frac{h_{2}^{2} h_{3}^{2}}{h_{1}^{2} h_{3}^{3}+h_{2}^{2} h_{3}^{3}+h_{1}^{5}}, a_{2}=\frac{h_{1}^{2} h_{3}^{2}}{h_{1}^{2} h_{3}^{3}+h_{2}^{2} h_{3}^{3}+h_{1}^{5}}, \\
& a_{3}=\frac{h_{1}^{5}}{h_{1}^{2} h_{3}^{3}+h_{2}^{2} h_{3}^{3}+h_{1}^{5}}
\end{aligned}
$$

$$
P L \_S_{3}^{\prime \prime}=\frac{h_{1}^{2} h_{2}^{4} h_{3}^{6}+h_{1}^{4} h_{2}^{2} h_{3}^{6}+h_{1}^{10} h_{3}^{2}}{\left(h_{1}^{2} h_{2}^{3}+h_{2}^{2} h_{3}^{3}+h_{1}^{5}\right)^{2}}
$$

- Our strategy: The three-user factor forms are given by (36), and the target is indicated by (37).

$$
\begin{aligned}
& a_{1}=\frac{h_{2} h_{3}}{h_{1} h_{2}+h_{2} h_{3}+h_{1} h_{3}}, a_{2}=\frac{h_{1} h_{3}}{h_{1} h_{2}+h_{2} h_{3}+h_{1} h_{3}}, \\
& a_{3}=\frac{h_{1} h_{2}}{h_{1} h_{2}+h_{2} h_{3}+h_{1} h_{3}}
\end{aligned}
$$

Our_S $S_{3}^{\prime \prime}=\frac{h_{1}^{2} h_{2}^{2} h_{3}^{2}}{\frac{1}{3}\left(h_{1} h_{2}+h_{2} h_{3}+h_{1} h_{3}\right)^{2}}$

\subsubsection{Comparison between power law strategy and our strategy}

Due to the complex mathematical form of the power law strategy, we perform the following comparison in the approximate forms. Within crowds of VLC UDN, an optical attocell is much smaller than domestic femtocells or WiFi coverage, so that we make an approximate numerical calculation $h_{1}^{6} \approx h_{2}^{4} h_{3}^{2}$ under the equal power counting. After we substitute (4) into (35) and (37), (38) and (39) are derived, where $\theta=(m+3)$ is positive according to (2) and $x, y, z$ are as indicated in (40). 


$$
\begin{aligned}
P L_{-} S_{3}^{\prime \prime} & \approx\left(\rho(\theta-2) L^{\theta-2}\right)^{2} \cdot \frac{x^{8 \theta}+y^{6 \theta} z^{2 \theta}+y^{4 \theta} z^{4 \theta}}{\left(x^{5 \theta}+x^{3 \theta} y^{2 \theta}+y^{2 \theta} z^{3 \theta}\right)^{2}} \\
O u r_{-} S_{3}^{\prime \prime} & =\frac{3\left(\rho(\theta-2) L^{\theta-2}\right)^{2}}{\left(x^{\theta}+y^{\theta}+z^{\theta}\right)^{2}} \\
x & =\sqrt{r_{1}^{2}+L^{2}}, y=\sqrt{r_{2}^{2}+L^{2}}, z=\sqrt{r_{3}^{2}+L^{2}}
\end{aligned}
$$

Due to the NOMA-VLC pre-conditions of (6) and (7), the relationship among $x, y$, and $z$ can be inferred as (41) with $\theta>0$. Note that the typical indoor scenario of a VLC single cell is that a VLC AP on the room ceiling covers a circle for communication, as shown in Fig. 1. Indoor architecture standards [37-39] indicate that the height between overhead illuminants on the ceiling and user receivers should exceed $1 \mathrm{~m}$. (41) indicates that the radii of user positions are distributed between zero and maximum available communication radius by FOV. Hence, (41) is the defined domain of the radius, which can be adapted to a broad class of typical indoor scenarios.

$$
\begin{gathered}
h_{1}<h_{2}<h_{3} \Leftrightarrow 0<r_{3}<r_{2}<r_{1}<r_{\max } \\
\Leftrightarrow 1<L<z<y<x<\sqrt{r_{\max }^{2}+L^{2}}
\end{gathered}
$$

(1) General NOMA-VLC user scenario: inequality condition To compare Our_S $S_{3}^{\prime \prime}$ with $P L \_S_{3}^{\prime \prime}$, the general VLC scenario is applied, where the VLC channel gains of each user follow the strict inequality $h_{1}<h_{2}<h_{3} \Rightarrow x>y>$ $z$. Due to the unavailability of equivalency among $h_{1}, h_{2}$ and $h_{3}$, the ratio and mean value inequality methods are exploited in (42) and (43). Discussing whether the ratio Our_S $S_{3}^{\prime \prime} / P L \_S_{3}^{\prime \prime}$ is greater than 1 is our goal.

$$
\begin{aligned}
\frac{O u r_{-} S_{3}^{\prime \prime}}{P L \_S_{3}^{\prime \prime}} & =\frac{3\left(x^{5 \theta}+x^{3 \theta} y^{2 \theta}+y^{2 \theta} z^{3 \theta}\right)^{2}}{\left(x^{\theta}+y^{\theta}+z^{\theta}\right)^{2} \cdot\left(x^{8 \theta}+y^{6 \theta} z^{2 \theta}+y^{4 \theta} z^{4 \theta}\right)} \\
& >\frac{\left(x^{5 \theta}+x^{3 \theta} y^{2 \theta}+y^{2 \theta} z^{3 \theta}\right)^{2}}{\left(x^{2 \theta}+y^{2 \theta}+z^{2 \theta}\right) \cdot\left(x^{8 \theta}+y^{6 \theta} z^{2 \theta}+y^{4 \theta} z^{4 \theta}\right)} \\
& \triangleq \frac{Q_{\text {Our_S } S_{3}^{\prime \prime}}}{Q_{P L \_} S_{3}^{\prime \prime}}
\end{aligned}
$$

We denote the molecule and denominator of (43) as (44) and (45), respectively. It is evident that $Q_{O u r_{-} S_{3}^{\prime \prime}}^{1}$ is equal to $Q_{P L_{-} S_{3}^{\prime \prime}}^{1}$.

$$
\begin{aligned}
& Q_{O u r_{-} S_{3}^{\prime \prime}}=\left(x^{5 \theta}+x^{3 \theta} y^{2 \theta}+y^{2 \theta} z^{3 \theta}\right)^{2} \\
& =\underbrace{\left(x^{10 \theta}+x^{8 \theta} y^{2 \theta}+y^{4 \theta} z^{6 \theta}\right)}_{Q_{\text {Our_ } S_{3}^{\prime \prime}}^{1}}
\end{aligned}
$$

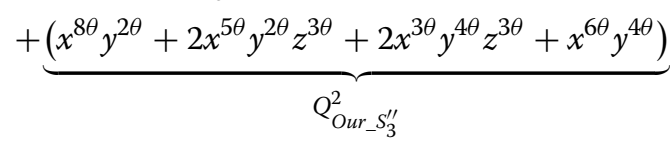

$$
\begin{aligned}
& Q_{P L_{-} S_{3}^{\prime \prime}}=\left(x^{2 \theta}+y^{2 \theta}+z^{2 \theta}\right) \cdot\left(x^{8 \theta}+y^{6 \theta} z^{2 \theta}+y^{4 \theta} z^{4 \theta}\right) \\
& =\underbrace{\left(x^{10 \theta}+x^{8 \theta} y^{2 \theta}+y^{4 \theta} z^{6 \theta}\right)}_{Q_{P L_{-} s_{3}^{\prime \prime}}^{1}} \\
& +\underbrace{\left(x^{8 \theta} z^{2 \theta}+2 y^{6 \theta} z^{4 \theta}+x^{2 \theta} y^{6 \theta} z^{2 \theta}+x^{2 \theta} y^{4 \theta} z^{4 \theta}+y^{8 \theta} z^{2 \theta}\right)}_{Q_{P L_{-} S_{3}^{\prime \prime}}^{2}}
\end{aligned}
$$

When $Q_{O u r_{-} S_{3}^{\prime \prime}}^{1}=Q_{P L_{-} S_{3}^{\prime \prime}}^{1}$, we should illustrate the relationship $Q_{O u r_{-} S_{3}^{\prime \prime}}^{2}>Q_{P L \_S_{3}^{\prime \prime}}^{2}$ further by analytic solutions. Using the difference between $Q_{\text {Our_S } S_{3}^{\prime \prime}}^{2}$ and $Q_{P L_{-} S_{3}^{\prime \prime}}^{2}$, we should prove that the difference (46) is positive. Based on (41), it is clear that $Q_{(1)}^{2}>0$ and $Q_{(2)}^{2}>0$ in (46).

- If $Q_{(3)}^{2} \geq 0$, the difference $\left(Q_{O u r_{-} S_{3}^{\prime \prime}}^{2}-Q_{P L_{-} S_{3}^{\prime \prime}}^{2}\right)$ should be positive.

- If $Q_{(3)}^{2}<0$, due to the relationship $x^{8 \theta}>x^{2 \theta} y^{4 \theta} z^{2 \theta}$, we can establish (47) to remain the positive.

$$
\begin{aligned}
Q_{\text {Our_ } S_{3}^{\prime \prime}}^{2}-Q_{P L_{-} S_{3}^{\prime \prime}}^{2}= & \underbrace{2 y^{2 \theta} z^{3 \theta}\left(x^{5 \theta}-y^{4 \theta} z^{\theta}\right)+y^{4 \theta}\left(x^{6 \theta}-y^{4 \theta} z^{2 \theta}\right)}_{Q_{(1)}^{2}} \\
& +\underbrace{x^{8 \theta}\left(y^{2 \theta}-z^{2 \theta}\right)}_{Q_{(2)}^{2}}+\underbrace{x^{2 \theta} y^{4 \theta} z^{2 \theta}\left(2 x^{\theta} z^{\theta}-z^{2 \theta}-y^{2 \theta}\right)}_{Q_{(3)}^{2}}
\end{aligned}
$$

$$
\begin{aligned}
Q_{\text {Our_ } S_{3}^{\prime \prime}}^{2}-Q_{P L_{-} S_{3}^{\prime \prime}}^{2}= & Q_{(1)}^{2}+Q_{(2)}^{2}+Q_{(3)}^{2} \\
> & Q_{(1)}^{2}+x^{8 \theta}\left(y^{2 \theta}-z^{2 \theta}\right) \\
& +x^{8 \theta}\left(2 x^{\theta} z^{\theta}-z^{2 \theta}-y^{2 \theta}\right) \\
= & Q_{(1)}^{2}+2 x^{8 \theta}\left(x^{\theta}-z^{\theta}\right) z^{\theta} \\
> & 0
\end{aligned}
$$

Based on (42)-(47), conclusion (48) can be drawn.

$$
\frac{O u r_{\_} S_{3}^{\prime \prime}}{P L \_S_{3}^{\prime \prime}}>\frac{Q_{O u r_{-} S_{3}^{\prime \prime}}^{1}+Q_{O u r_{-} S_{3}^{\prime \prime}}^{2}}{Q_{P L_{-} S_{3}^{\prime \prime}}^{1}+Q_{P L \_}^{2} S_{3}^{\prime \prime}}>1
$$

(2) Special NOMA-VLC user scenario: local equality condition In special VLC scenarios, the 3-user channel 
gains may be equal in pairs as indicated by (49), and (43) may not be available.

$$
\left\{\begin{array}{l}
0<h_{1}=h_{2}<h_{3} \Rightarrow x=y>z>1 \\
0<h_{1}<h_{2}=h_{3} \Rightarrow x>y=z>1
\end{array}\right.
$$

- $x=y>z: \ln (50)$, it is obvious that part $_{1}^{\text {Our }}>$ part $_{1}^{\text {PLS }}$, part $_{2}^{\text {Our }}>$ part $_{2}^{\text {PLS }}$ and
part $_{3}^{\text {Our }}>$ part $_{3}^{\text {PLS }}$ because of (49) and $\theta>0$.

$$
\begin{aligned}
& \frac{O u r_{-} S_{3}^{\prime \prime}}{P L_{-} S_{3}^{\prime \prime}}=\frac{3\left(2 x^{5 \theta}+x^{2 \theta} z^{3 \theta}\right)^{2}}{\left(2 x^{\theta}+z^{\theta}\right)^{2} \cdot\left(x^{8 \theta}+x^{6 \theta} z^{2 \theta}+x^{4 \theta} z^{4 \theta}\right)} \\
& \underbrace{\left(12 x^{10 \theta}\right)}_{\text {part } t_{1}^{\text {Our }}}+\underbrace{\left(9 x^{7 \theta} z^{3 \theta}\right)}_{\text {part } 2_{2}^{\text {Our }}} \\
& =\underbrace{\frac{\text { part }_{1}^{\text {Our }}}{\left(4 x^{10 \theta}+4 x^{8 \theta} z^{2 \theta}+4 x^{9 \theta} z^{\theta}\right)}}_{\text {part }}+\underbrace{\left(4 x_{2}^{\text {PLS }}\right.}_{\text {part }}
\end{aligned}
$$

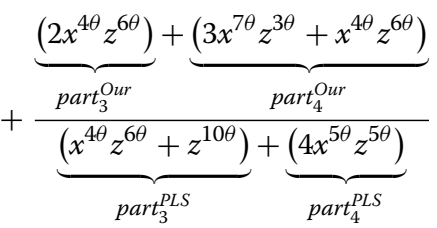

$$
\begin{aligned}
\operatorname{part}_{4}^{\text {Our }}-\operatorname{part}_{4}^{\text {PLS }} & =3 x^{7 \theta} z^{3 \theta}+x^{4 \theta} z^{6 \theta}-4 x^{5 \theta} z^{5 \theta} \\
& =x^{4 \theta} z^{3 \theta}\left(x^{2 \theta}-z^{2 \theta}+3 x^{\theta} z^{\theta}+2 x^{2 \theta}\right)\left(x^{\theta}-z^{\theta}\right) \\
& >0
\end{aligned}
$$

To demonstrate the hypothesis that

Our_S $S_{3}^{\prime \prime}>P L \_S^{\prime \prime}$, the condition holding

part $_{4}^{\text {Our }}>$ part $_{4}^{\text {PLS }}$ should be valid. Due to the validity of (51), part $_{4}^{\text {Our }}>$ part $_{4}^{\text {PLS }}$ is true, and our strategy is better than the power law strategy in this special case.

- $x>y=z: \operatorname{In}(52)$, it is obvious that

part $_{5}^{\text {Our }}=$ part $_{5}^{\text {PLS }}$, part $_{6}^{\text {Our }}>$ part $_{6}^{\text {PLS }}$, part $_{7}^{\text {Our }}>$ part $_{7}^{\text {PLS }}$, and part Our $_{8}>$ part $_{8}^{\text {PLS }}$. The relationship between part $_{9}^{\text {Our }}$ and part ${ }_{9}^{\text {PLS }}$ can be given by (53). Hence, in this special case, our strategy is better than the power law strategy.

$$
\begin{aligned}
& \frac{O u r_{-} S_{3}^{\prime \prime}}{P L_{-} S_{3}^{\prime \prime}}=\frac{3\left(x^{5 \theta}+x^{3 \theta} y^{2 \theta}+y^{5 \theta}\right)^{2}}{\left(x^{\theta}+2 y^{\theta}\right)^{2} \cdot\left(x^{8 \theta}+2 y^{8 \theta}\right)}
\end{aligned}
$$

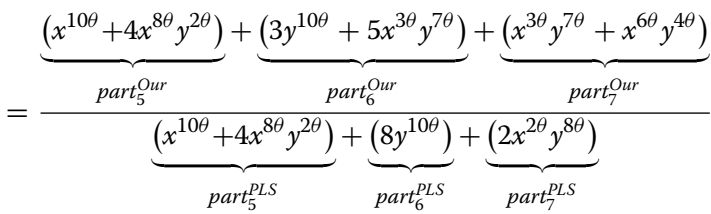

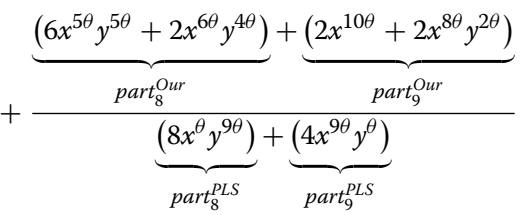

$$
\begin{aligned}
\operatorname{part}_{9}^{\text {Our }}-\text { part }_{9}^{\text {PLS }} & =2 x^{10 \theta}+2 x^{8 \theta} y^{2 \theta}-4 x^{9 \theta} y^{\theta} \\
& =2\left(x^{\theta}-y^{\theta}\right)^{2} \cdot x^{8 \theta}>0
\end{aligned}
$$

\section{Results and discussion}

\subsection{The SNR distribution in a single VLC cell}

As indicated in Table 1, the relevant simulation parameters are as reported in $[1,6,10,11]$. If the room is $6 \mathrm{~m}$ long and $6 \mathrm{~m}$ wide, Fig. 2 gives the signal-to-noise ratio (SNR) distribution from a single LED at height of $2.5 \mathrm{~m}$ located in the centre of the room. Figure 2 indicates the inhomogeneous received signal strength of each sub-area size when $\Phi_{1 / 2}=60^{\circ}$ and $\Psi_{F O V}=60^{\circ}$. In Fig. 2, it can be envisioned that edge users may exist in sub-areas of the poor communication quality region with the growing number of total users in a single cell.

\subsection{Comparisons of two- and three-user cases}

In this subsection, we focus on the typical application scenario of VLC networks supporting two or three indoor family users. According to the parameters shown in Fig. 2 and Monte Carlo simulations performed at each angle, Fig. 3 compares two NOMA-VLC GRPA strategies with different $\Phi_{1 / 2}$ and fixed $\Psi_{F O V}$. If the index of the average user data rate (AUDR) is evaluated, Fig. 3 shows that our GRPA strategy has a better AUDR than does the power law strategy.

When $\Psi_{F O V}=60^{\circ}$, each multi-access method may yield the best performance at approximately $\Phi_{1 / 2}=$ $35^{\circ}$ in two- and three-user cases. As $\Phi_{1 / 2}$ increases, the performance of each multi-access method increases first and then decreases with $\Phi_{1 / 2}>35^{\circ}$. For the two power control strategies, Fig. 3 shows four curves of multi-access methods exhibiting similar increasingdecreasing trends, which indicates that not only signal power allocation but also illumination parameters can affect the performance of per capita throughput in a VLC network.

According to (4), the conclusion regarding the effect on AUDR in Fig. 3 based on the original target can be explained by our alternative target as well. If $\Psi_{F O V}$ is fixed, then $g\left(\psi_{k}\right)$ and $\rho$ are fixed. When $\Phi_{1 / 2}$ increases from $10^{\circ}$ to $60^{\circ}$, the order of Lambertian emission $m$ decreases, as indicated by (2). In addition, regardless of factor values such as $a_{k}$ and $\tau_{k}$ based on either strategy, the user data rate retains a monotonicity similar to that shown in Fig. 3. Therefore, the following realistic assumptions can be reached.

- $\Phi_{1 / 2}$ is the unique independent variable of Lambertian emission order $m$.

- $\log _{2}(\bullet)$ will not transform the increasing monotonicity of $\sum_{k=1}^{K}\left(\gamma h_{k} a_{k}\right)^{2}$ into the decreasing monotonicity. 
Table 1 Reference parameters

\begin{tabular}{lll}
\hline Symbol & Name & Value \\
\hline$\gamma$ & Optical-electrical conversion efficiency & $0.5(\mathrm{~A} / \mathrm{W})$ \\
$a_{1}, a_{2}, \cdots, a_{K}$ & GRPA factor & $0<a_{K}<\cdots<a_{2}<a_{1}<1$ \\
$\Psi_{\text {FOV }}$ & Angle field of vision & $60^{\circ}$ \\
$\Phi_{1 / 2}$ & Semi-angle & $60^{\circ}$ \\
$A$ & Area of PD & $10^{-4}\left(\mathrm{~m}^{2}\right)$ \\
$T_{\text {filter }}$ & Gain of an optical filter & 1 \\
$n$ & Refractive index & 1.5 \\
$g\left(\psi_{k}=\Psi_{\text {FOV }}\right)$ & Gain of an optical concentrator & 3 \\
$\rho$ & A $\cdot T_{\text {filter }} \cdot g\left(\psi_{k}\right) / 2 \pi$ & $\rho \approx 5 \times 10^{-5}$ \\
$m=-1 / \log _{2}\left(\cos \left(\Phi_{1 / 2}=60^{\circ}\right)\right)$ & Order of Lambertian emission & 1 \\
$L$ & Height of the LED above receivers & $2.5(\mathrm{~m})$ \\
$N_{0}$ & Noise power spectral density & $10^{-19}\left(\mathrm{~A}^{2} / \mathrm{Hz}\right)$ \\
$B$ & Bandwidth of a single LED & $20(\mathrm{MHz})$ \\
$P T$ & Transmitted power of a single LED & $2(\mathrm{~W})$ \\
$R O O M$ & Room size & $6 \times 6\left(\mathrm{~m}^{2}\right)$ \\
\hline
\end{tabular}

- If a user located in the coverage of a VLC AP follows a uniform distribution, in polar coordinates, the radius of a point or user is determined from the density $f(r)=\frac{2 r_{k}}{\left(r_{\max }\right)^{2}}$, where $r_{k}$ is the radius of the $k$-th user and $r_{\max }$ is the maximum radius of available received coverage. Focusing on the two-user case and Fig. 2, we can choose two users from a sufficient number of users and $r_{\max }=3$, which is related to room size. The expectation average radius $\bar{r}_{k}$ of two users is approximately equal to the centroid of a sufficient number of users, as indicated by (54).

$$
\bar{r}_{k}=\frac{\int_{0}^{r_{\max }} f\left(r_{k}\right) \cdot r_{k} d r_{k}}{\int_{0}^{r_{\max }} f\left(r_{k}\right) d r_{k}}=\frac{2}{3} r_{\max }
$$

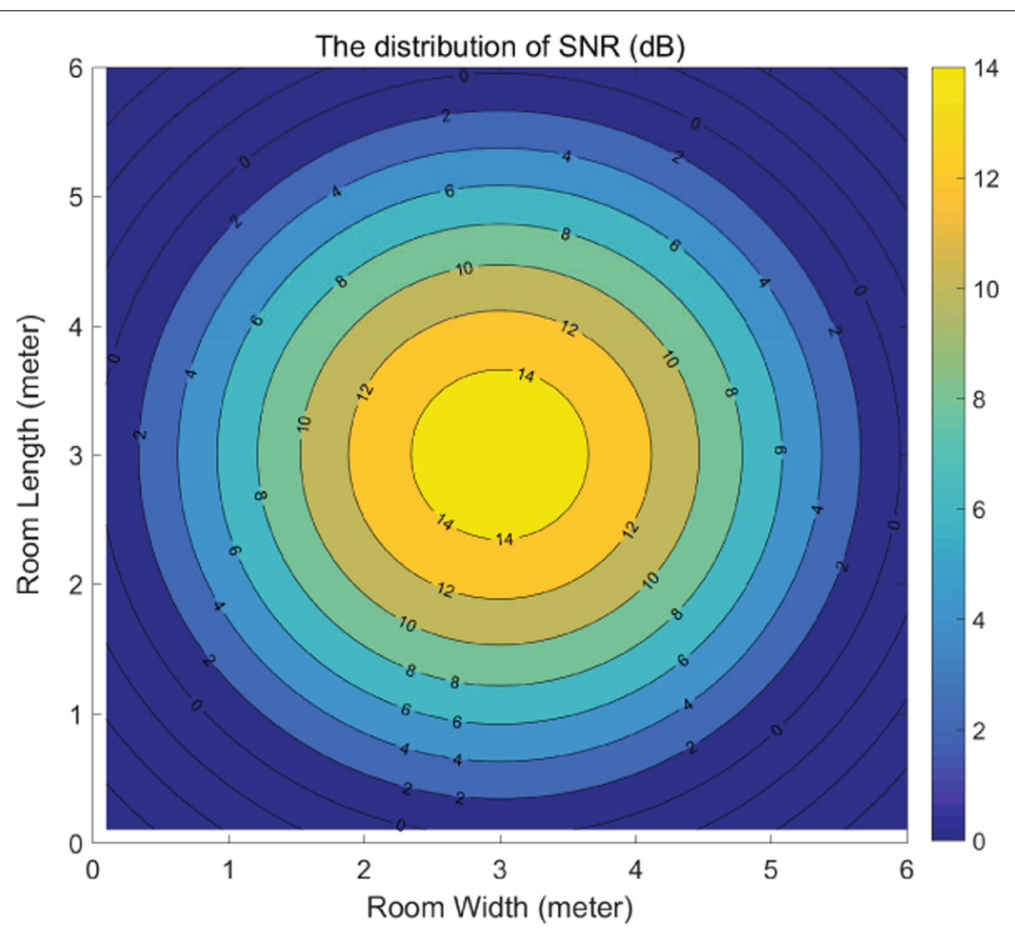

Fig. 2 The distribution of signal-to-noise ratio (SNR) 


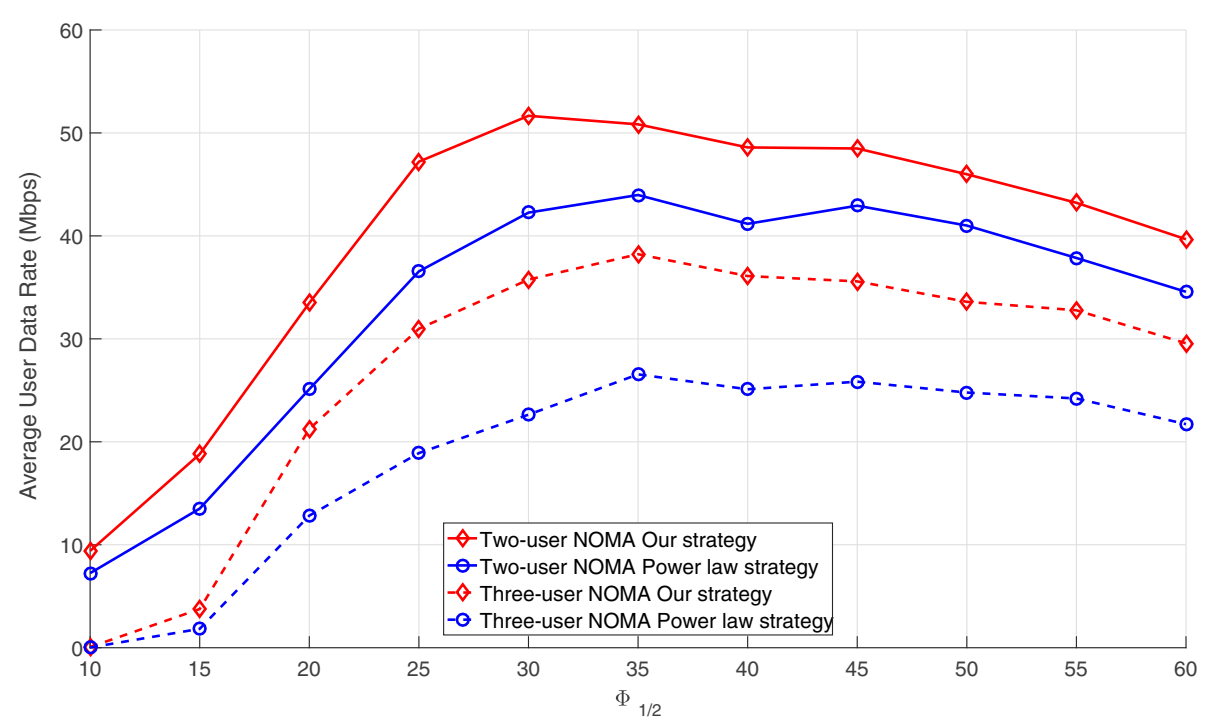

Fig. 3 The AUDR comparison in two- and three-user cases with $\Psi_{F O V}=60^{\circ}$

- Given $\rho, r_{k}$, fixed $\Psi_{F O V}$, and the definite values of power allocation factors, we focus on the relationship between $\Phi_{1 / 2}$ and the two-user data rate in NOMA-VLC networks, as indicated by (55).

- The parameters of our alternative target forms are the same as those of the original target in Fig. 3.

$$
\frac{\partial\left(1+\frac{\sum_{k=1}^{K}\left(\gamma h_{k} a_{k}\right)^{2}}{N_{0} B}\right)}{\partial m}=\left[\frac{\partial \sum_{k=1}^{K}\left(\gamma h_{k} a_{k}\right)^{2}}{\partial m}\right]=0
$$

$$
\begin{aligned}
\lim _{K \rightarrow+\infty, k \in K}\left(\frac{\partial \sum_{k=1}^{2}\left(\gamma h_{k} a_{k}\right)^{2}}{\partial m}\right) & \left.\approx \frac{\partial \mathrm{E}\left[\bar{h}_{k}^{2}\right]}{\partial m}\right|_{\mathrm{E}\left[\bar{r}_{k}\right],|k|=2} \\
& =\frac{2 \rho L^{m+1} \cdot \ln \left(e \cdot\left(\frac{L^{2}}{\bar{r}_{k}^{2}+L^{2}}\right)^{\frac{m+1}{2}}\right)}{\left(\bar{r}_{k}^{2}+L^{2}\right)^{\frac{m+3}{2}}}
\end{aligned}
$$$$
=0
$$

$$
m^{*}=\frac{2}{\ln \left(\frac{\bar{r}_{k}^{2}+L^{2}}{L^{2}}\right)}-1
$$

After we reduce the mathematical form of (55) to the twouser situation, (57) yields the extremal solution of $m$ based on (56). In our alternative bound, (56) and (57) can indicate that the user data rate grows in the left region of the extremum of $m^{*}$ and decreases in the right region of extremum of $m^{*}$ with fixed user number. If the parameters listed in Table 1 and $\bar{r}_{k}=\frac{2}{3} r_{\max }$ are given, $m^{*}=3.04$ and $\Phi_{1 / 2} \approx 37.2^{\circ}$ are obtained by the alternative target, in agreement with Fig. 3 for the original target.

On the other hand, this conclusion generated by our alternative target is similar to that reached in [10]. In [10], LEDs with a semi-angle of $35^{\circ}$ yielded nearly optimal performance with the GRPA of NOMA, and the extremum of the semi-angle increased with the number of users.

Hence, we prove that the monotonicity of Fig. 3 is the same as that indicated by the analysis of our alternative target. The monotonicity analysis indicates that the use of our lower bound is effective in solving the original problem with the suitable functional behaviour.

4.3 Comparisons between original and alternative targets According to the parameters shown in Fig. 2, the orthogonal frequency division multiple access (OFDMA) and NOMA strategies are analyzed in Fig. 4. The user positions are generated by a uniform distribution via Monte Carlo simulation. For OFDMA [40, 41] as (58) shows, when the transmitted power is fixed, the power allocation using a water-filling strategy and fixed power equipartition are denoted as (59) and (60), respectively.

$$
\begin{aligned}
\text { Rate }_{\text {OFDMA }}\left(h_{k}, \tau_{k}\right) & =\tau_{k} \cdot B \cdot \log _{2}\left(1+\frac{\left(\gamma \cdot h_{k} \cdot \tau_{k} \cdot P T\right)^{2}}{N_{0} \cdot B \cdot \tau_{k}}\right) \\
\tau_{k} & =\frac{h_{k}}{\sum_{k \in K} h_{k}} \\
\tau_{k}^{\prime} & =\frac{1}{\mid \text { Users } \mid}
\end{aligned}
$$




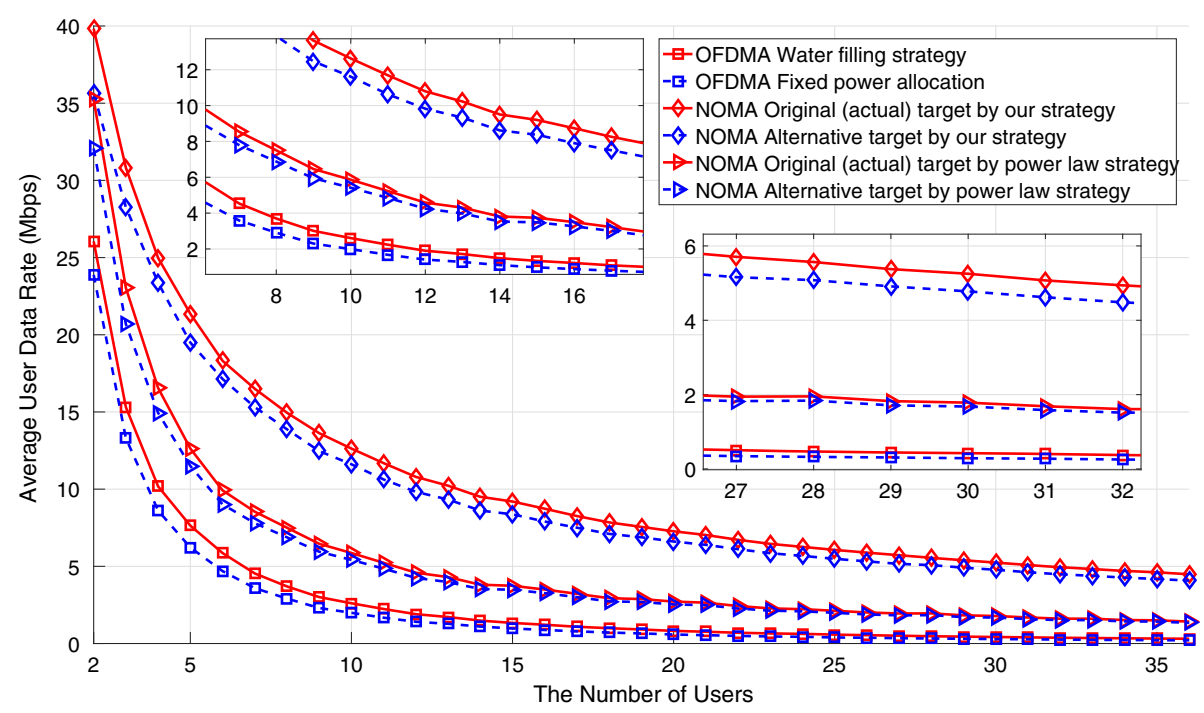

Fig. 4 The AUDR comparison among multi-access methods with $\Phi_{1 / 2}=\Psi_{\mathrm{FOV}}=60^{\circ}$

For NOMA strategies, this subsection discusses the approximate function property between the original target of (61) and our alternative target of (62). Figure 4 is obtained to analyze the performance difference between the two targets.

target $=\log _{2}\left[\left(1+\frac{\left(\gamma h_{K} a_{K}\right)^{2}}{N_{0} B}\right) \cdot \prod_{k=1}^{K-1}\left(1+\frac{\left(\gamma h_{k} a_{k}\right)^{2}}{N_{0} B+\sum_{i=k+1}^{K}\left(\gamma h_{k} a_{i}\right)^{2}}\right)\right]$

our alternative bound $=\log _{2}\left[\left(1+\frac{\sum_{k=1}^{K}\left(\gamma h_{k} a_{k}\right)^{2}}{N_{0} B}\right)\right]$

In Fig. 4, the convergence of two-target function monotonicity is confirmed. An alternative target is proposed in this paper because NOMA-VLC GRPA strategies are difficult to compare with respect to the original target. Figure 4 indicates that the revised mathematical form of the alternative target offers an appropriate lower bound for convenient of NOMA-VLC GRPA strategy comparisons.

\subsection{Comparisons with more users}

\subsubsection{Fixed $\Phi_{1 / 2}$ and $\Psi_{\text {FOV }}$}

In this subsection, we focus on the performance of the proposed strategy with multiple users (i.e., more than three users). With the increase in the number of users inside the room, the gap between NOMA and OMA decreases, as shown in Fig. 4. This behaviour implies that our strategy outperforms other methods involving multiple users as well. Our NOMA-VLC GRPA strategy achieves the greater variance around the horizontal ordinate point of four users compared with the power law strategy. In addition, (24) reveals a good asymptotic match, where the gap between the original and alternative targets decreases as the number of users increases, which is reflected in Fig. 4 as well. Therefore, Fig. 4 indicates that the NOMA is worth being popularized and that our GRPA strategy of NOMA is better than the power law strategy.

\subsubsection{Varied $\Phi_{1 / 2}$ and $\Psi_{\text {FOV }}$}

Based on the same parameters listed in Table 1, except $\Phi_{1 / 2}$ and $\Psi_{F O V}$, Fig. 5 is obtained by the variation of $\Phi_{1 / 2}$ and $\Psi_{F O V}$ to observe the influence of light angles on two GRPA strategies involving multiple users.

When $\Psi_{F O V}$ is fixed as shown in Fig. 5a, the decrease in $\Phi_{1 / 2}$ indicates that the shrinking coverage of a VLC cell leads to the light beamform concentration. In Fig. $5 \mathrm{a}$, when users are few, the AUDR with $\Phi_{1 / 2}=30^{\circ}$ is better than that with $\Phi_{1 / 2}=50^{\circ}$ in both our strategy and power law strategy. When the number of users grows, two performance curves of $\Phi_{1 / 2}=30^{\circ}$ and $\Phi_{1 / 2}=50^{\circ}$ in the power law strategy have the intersection point. Moreover, as the number of users increases, the AUDR in our strategy is better than that in the power law strategy, when $\Phi_{1 / 2}$ is distributed between $30^{\circ}$ and $50^{\circ}$. Even in the worst VLC channel environment with $\Phi_{1 / 2}=10^{\circ}$, our strategy maintains a narrow advantage over the power law strategy. When $\Phi_{1 / 2}$ is fixed as Fig. 5b shows, the increase in $\Psi_{F O V}$ causes a decrease in $g\left(\psi_{k}\right)$ and $h_{k}$ according to (3). The performance of our strategy and power law strategy may be reduced as $\Psi_{F O V}$ increases to $60^{\circ}$, and our strategy 


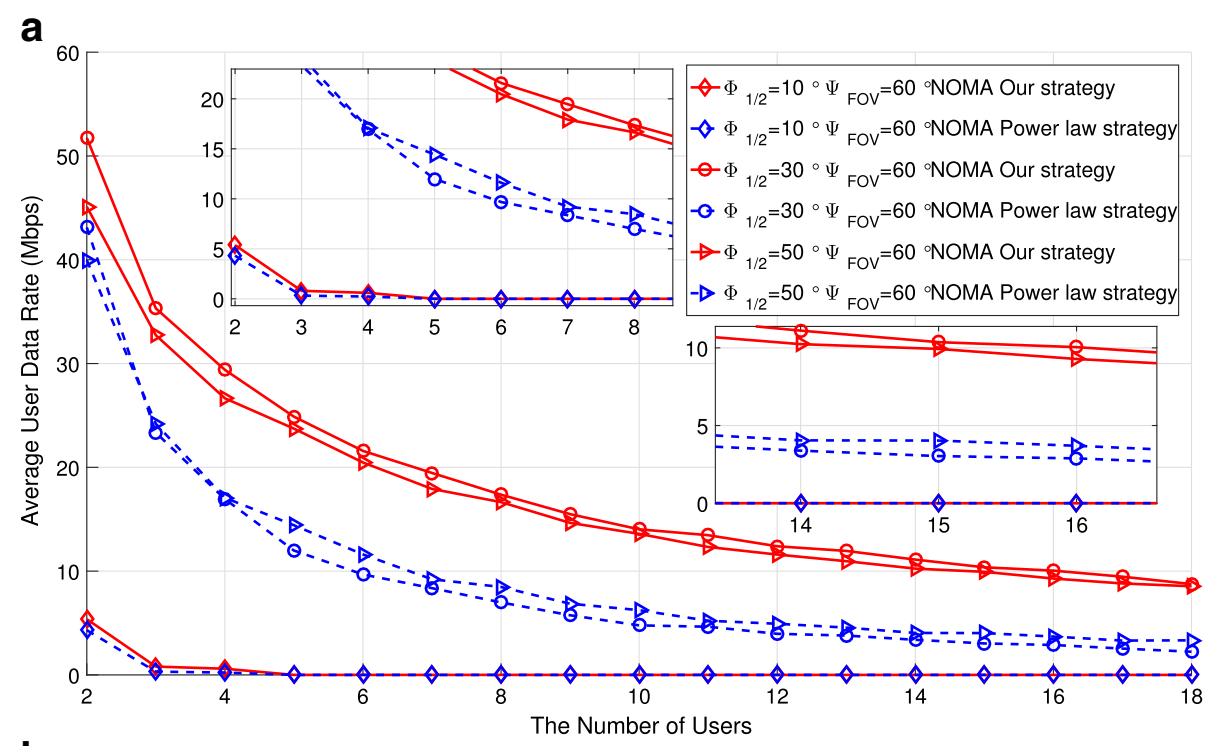

b

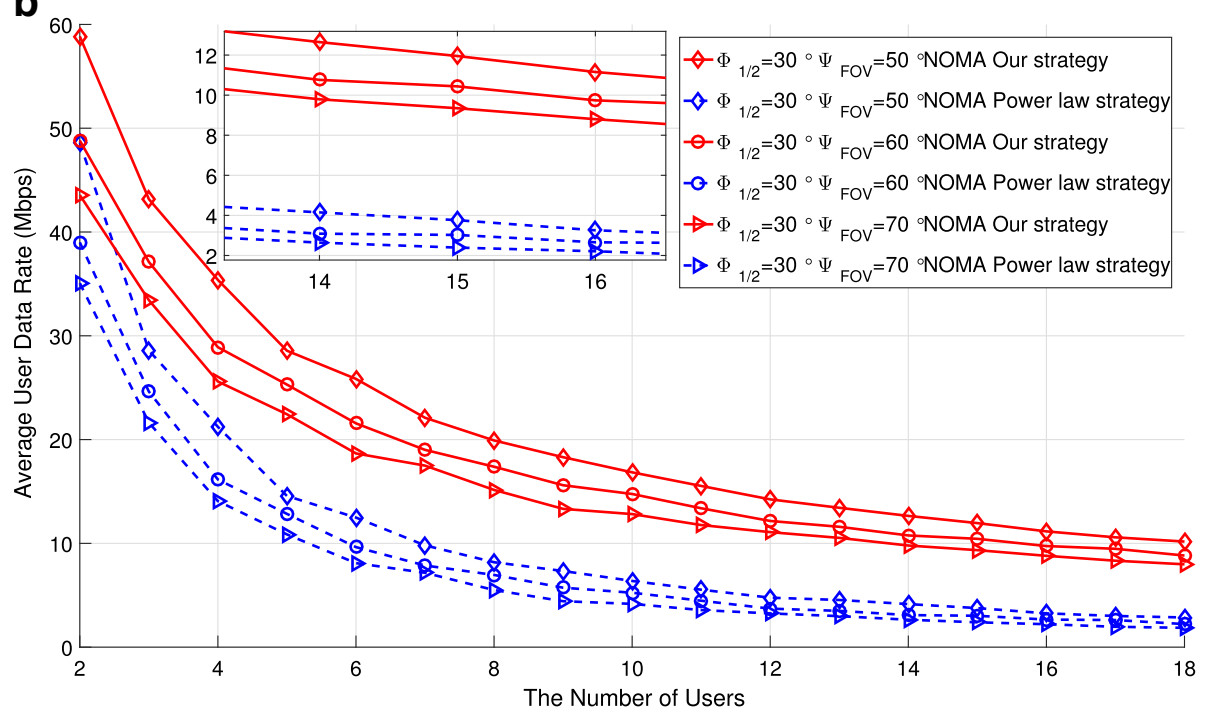

Fig. 5 The AUDR comparison between our strategy and power law strategy with an increasing number of users. a Comparison of NOMA-VLC GRPA strategies with fixed $\Psi_{F O V}$. b Comparison of NOMA-VLC GRPA strategies with fixed $\Phi_{1 / 2}$

outperforms the power law strategy with varied $\Phi_{1 / 2}$ and the same $\Psi_{F O V}$.

As shown in Figs. 4 and 5, our strategy yields suitable performance even under the worst receiving conditions, such as small angles of $\Phi_{1 / 2}$ and $\Psi_{F O V}$, with better AUDR performance than that of the power law strategy. The couple, i.e., $\Phi_{1 / 2}=30^{\circ}$ and $\Psi_{F O V}=40^{\circ}$, results in the highest AUDR via our strategy, indicating that any unilateral variety of $\Phi_{1 / 2}$ or $\Psi_{F O V}$ cannot sufficiently elevate the VLC network throughput.

\section{Conclusions}

Considering the inadequate processing capacity of VLC user receiver equipment, this paper dissects the solutions of the GRPA strategy for low complexity and convenient realization. The authors present a novel GRPA strategy of NOMA based on VLC channel gains and provide both analytic and numerical solutions. Comparisons between our strategy and power law strategy are performed based on an alternative bound proved by this paper. Two- and three-user cases show a better performance bound using our strategy, which is inextricably linked to VLC channel gains. Experimental results indicate that our strategy outperforms the previous strategy and OMA when the number of VLC users increases. The pre-condition of proof is established based on an illuminant height greater than $1 \mathrm{~m}$, which is applicable to broad indoor scenarios. In addition, both light angle parameters and power 


\section{allocation may affect the performance of NOMA in VLC networks, which deserves further research involving joint optimization.}

\begin{abstract}
Abbreviations
AP: Access point; AUDR: Average user data rate; FOV: Field of vision; GRPA: Gain ratio power allocation; IM/DD: Intensity modulation and direct detection; ISI: Inter-symbol interference; ISI-free: Inter-symbol interference free; LED: Light-emitting diode; LiFi: Light fidelity; LOS: Line of sight; NOMA: Non-orthogonal multiple access; OFDMA: Orthogonal frequency division multiple access; OMA: Orthogonal multiple access; OOK: On-off keying; Ours: Our strategy; OWC: Optical wireless communication; PD: Photo diode; PLS: Power law strategy; QoS: Quality of service; RF: Radio frequency; SIC: Successive interference cancellation; SINR: Signal interference plus noise ratio; SNR: Signal to noise ratio; UDN: Ultra dense network; VLC: Visible light communication; WiFi: Wireless fidelity; WLAN: Wireless local area network
\end{abstract}

\section{Acknowledgements}

This work was supported by the National Natural Science Foundation of China (No.61671477). The authors wish to acknowledge the valuable suggestions of Dr. Yanqun Tang, Wan Tian, M.Phil., Xiangwei Bai, M.Phil., and Dr. Xiaoyi Liu.

\section{Funding}

Funding for this study is from the National Natural Science Foundation of China (No.61671477).

\section{Authors' contributions}

We authors proposed the scheme of this paper. ST conducted the detailed derivations associated with performance analysis. HY, QL, and YT polished the article. All authors have read and approved the final manuscript.

\section{Authors' information}

Siyu Tao received his B.S. degree from the Beijing Institute of Technology in 2013. From 2013 to 2016, he pursued an M.S. degree from the National Digital Switching System Engineering and Technological Center (NDSC). He is currently studying for his doctorate at the NDSC. His research interests include visible light communication networking and network protocol reverse engineering.

Hongyi Yu received his Ph.D. from Xidian University in 1998. He is currently a professor at the NDSC, and his research interests include visible light communication, wireless communication and signal processing. Qing Li received her M.S. degree from the NDSC in 2003 and her Ph.D. from the NDSC in 2009. She is currently an associate professor working on visible light communication networking and network protocol reverse engineering. Yanqun Tang received his Ph.D. from the National University of Defense Technology in 2013. He is currently a university lecturer at the NDSC working on the physical layer security of wireless communication and visible light communication.

\section{Competing interests}

The authors declare that they have no competing interests.

\section{Publisher's Note}

Springer Nature remains neutral with regard to jurisdictional claims in published maps and institutional affiliations.

Received: 2 January 2018 Accepted: 25 May 2018

Published online: 15 June 2018

\section{References}

1. H Haas, L Yin, Y Wang, C Chen, What is LiFi? J. Light. Technol. 34(6), 1533-1544 (2016). https://doi.org/10.1109/JLT.2015.2510021

2. S Chen, F Qin, B Hu, X Li, Z Chen, J Liu, in User-Centric Ultra-Dense Networks for 5G. User-centric ultra-dense networks for 5G (Springer, German, 2018), pp. 8-9. https://doi.org/10.1007/978-3-319-61201-0_1

3. EE Tsiropoulou, P Vamvakas, S Papavassiliou, Supermodular game-based distributed joint uplink power and rate allocation in two-tier femtocell networks. IEEE Trans. Mob. Comput. 16(9), 2656-2667 (2017). https://doi. org/10.1109/TMC.2016.2622263
4. EE Tsiropoulou, P Vamvakas, S Papavassiliou, Resource allocation in next-generation broadband wireless access networks. (IGI Global, USA, 2017), pp. 210-246

5. S Islam, M Zeng, OA Dobre, K-S Kwak, Resource allocation for downlink NOMA systems: key techniques and open issues. IEEE Wirel. Commun. 25(2), 40-47 (2018). https://doi.org/10.1109/MWC.2018.1700099

6. TKomine, M Nakagawa, Fundamental analysis for visible-light communication system using led lights. IEEE Trans. Consum. Electron. 50(1), 100-107 (2004). https://doi.org/10.1109/TCE.2004.1277847

7. R Jiang, Q Wang, H Haas, Z Wang, Joint user association and power allocation for cell-free visible light communication networks. IEEE J. Sel. Areas Commun. 36(1), 136-148 (2018). https://doi.org/10.1109/JSAC. 2017.2774400

8. H Haas, High-speed wireless networking using visible light. SPIE Newsroom. 10(2.1201304), 1-3 (2013). https://doi.org/10.1117/2. 1201304.004773

9. X Zhang, Q Gao, C Gong, Z Xu, User grouping and power allocation for NOMA visible light communication multi-cell networks. IEEE Commun. Lett. 21(4), 777-780 (2017). https://doi.org/10.1109/LCOMM.2016. 2642921

10. L Yin, WO Popoola, X Wu, H Haas, Performance evaluation of non-orthogonal multiple access in visible light communication. IEEE Trans. Commun. 64(12), 5162-5175 (2016). https://doi.org/10.1109/ TCOMM.2016.2612195

11. H Marshoud, VM Kapinas, GK Karagiannidis, S Muhaidat, Non-orthogonal multiple access for visible light communications. IEEE Photon. Technol. Lett. 28(1), 51-54 (2016). https://doi.org/10.1109/LPT.2015.2479600

12. K Lai, J Lei, L Wen, G Chen, W Li, P Xiao, Secure transmission with randomized constellation rotation for downlink sparse code multiple access system. IEEE Access. 6, 5049-5063 (2018). https://doi.org/10.1109/ ACCESS.2017.2772259

13. EE Tsiropoulou, I Gialagkolidis, P Vamvakas, S Papavassiliou, in International Conference on Ad-Hoc Networks and Wireless. Resource allocation in visible light communication networks: NOMA vs OFDMA transmission techniques (Springer, Cham, 2016), pp. 32-46. https://doi. org/10.1007/978-3-319-40509-4_3

14. SR Islam, N Avazov, OA Dobre, K-S Kwak, Power-domain non-orthogonal multiple access (NOMA) in 5G systems: Potentials and challenges. IEEE Commun. Surv. Tutor. 19(2), 721-742 (2017). https://doi.org/10.1109/ COMST.2016.2621116

15. Z Yang, W Xu, Y Li, Fair non-orthogonal multiple access for visible light communication downlinks. IEEE Wirel. Commun. Lett. 6(1), 66-69 (2017). https://doi.org/10.1109/LWC.2016.2631471

16. TV Pham, AT Pham, in Globecom Workshops (GC Wkshps), 2015 IEEE. Maxmin fairness and sum-rate maximization of MU-VLC local networks (IEEE, USA, 2015), pp. 1-6. https://doi.org/10.1109/GLOCOMW.2015.7414133

17. L Lei, D Yuan, CK Ho, S Sun, Power and channel allocation for non-orthogonal multiple access in $5 \mathrm{G}$ systems: Tractability and computation. IEEE Trans. Wirel. Commun. 15(12), 8580-8594 (2016) https://doi.org/10.1109/TWC.2016.2616310

18. B Di, L Song, Y Li, Sub-channel assignment, power allocation, and user scheduling for non-orthogonal multiple access networks. IEEE Trans. Wirel. Commun. 15(11), 7686-7698 (2016). https://doi.org/10.1109/TWC. 2016.2606100

19. F Fang, H Zhang, J Cheng, VC Leung, Energy-efficient resource allocation for downlink non-orthogonal multiple access network. IEEE Trans. Commun. 64(9), 3722-3732 (2016). https://doi.org/10.1109/TCOMM. 2016.2594759

20. M Kashef, M Ismail, M Abdallah, K Qaraqe, E Serpedin, in Signal Processing Conference (EUSIPCO), 2015 23rd European. Power allocation for maximizing energy efficiency of mixed RF/VLC wireless networks (IEEE, 2015), pp. 1441-1445. https://doi.org/10.1109/EUSIPC0.2015.7362622

21. Q-V Pham, CS Hong, in Korea Software Conference. Power control for harmonic utility in non-orthogonal multiple access based visible light communications, (2017), pp. 1-3

22. R Mitra, V Bhatia, Precoded Chebyshev-NLMS based pre-distorter for nonlinear led compensation in NOMA-VLC. IEEE Trans. Commun. 65(11), 4845-4856 (2017). https://doi.org/10.1109/TCOMM.2017.2736548

23. Y Yapici, I Guvenc, Non-orthogonal multiple access for mobile VLC networks with random receiver orientation. arXiv preprint arXiv:1801.04888, 1-6 (2018). 1801.04888 
24. MF Hanif, Z Ding, T Ratnarajah, GK Karagiannidis, A minorization-maximization method for optimizing sum rate in the downlink of non-orthogonal multiple access systems. IEEE Trans. Signal Process. 64(1), 76-88 (2016). https://doi.org/10.1109/TSP.2015.2480042

25. H Shen, Y Wu, W Xu, C Zhao, Optimal power allocation for downlink two-user non-orthogonal multiple access in visible light communication. J. Commun. Inf. Netw. 2(4), 57-64 (2017). https://doi.org/10.1007/s41650017-0037-3

26. C Chen, WD Zhong, H Yang, P Du, On the performance of MIMO-NOMA based visible light communication systems. IEEE Photon. Technol. Lett. PP(99), 307-310 (2017). https://doi.org/10.1109/LPT.2017.2785964

27. L Lei, $P$ Värbrand, D Yuan, On power minimization for non-orthogonal multiple access (noma). IEEE Commun. Lett. 20(12), 2458-2461 (2016). https://doi.org/10.1109/LCOMM.2016.2606596

28. S Shi, L Yang, H Zhu, Outage balancing in downlink nonorthogonal multiple access with statistical channel state information. IEEE Trans. Wirel. Commun. 15(7), 4718-4731 (2016). https://doi.org/10.1109/TWC. 2016.2544922

29. Z Ding, X Lei, GK Karagiannidis, R Schober, J Yuan, V Bhargava, A survey on non-orthogonal multiple access for $5 \mathrm{G}$ networks: Research challenges and future trends. IEEE J. Sel. Areas Commun. 35(10), 2181-2195 (2017). https://doi.org/10.1109/JSAC.2017.2725519

30. H Shen, Y Deng, W Xu, C Zhao, Rate maximization for downlink multiuser visible light communications. IEEE Access. 4(99), 6567-6573 (2017). https://doi.org/10.1109/ACCESS.2016.2614598

31. A Lapidoth, SM Moser, MA Wigger, On the capacity of free-space optical intensity channels. IEEE Trans. Inf. Theory. 55(10), 4449-4461 (2009). https://doi.org/10.1109/TIT.2009.2027522

32. X Li, R Zhang, L Hanzo, Cooperative load balancing in hybrid visible light communications and wifi. IEEE Trans. Commun. 63(4), 1319-1329 (2015). https://doi.org/10.1109/TCOMM.2015.2409172

33. X Li, F Jin, R Zhang, J Wang, Users first: User-centric cluster formation for interference-mitigation in visible-light networks. IEEE Trans. Wirel. Commun. 15(1), 39-53 (2016). https://doi.org/10.1109/TWC.2015.2466539

34. J Zhou, W Zhang, On the capacity of bandlimited optical intensity channels with Gaussian noise. IEEE Trans. Commun. 65(6), 2481-2493 (2017). https://doi.org/10.1109/TCOMM.2017.2669966

35. A Benjebbovu, A Li, Y Saito, Y Kishiyama, A Harada, T Nakamura, in 2013 IEEE Globecom Workshops (GC Wkshps). System-level performance of downlink NOMA for future LTE enhancements, (2013), pp. 66-70. https:// doi.org/10.1109/GLOCOMW.2013.6824963

36. TXJG Xiang, A low computational complexity power allocation algorithm for non-orthogonal multiple access systems. Appl. Electron. Tech. 43(4), 126-128 (2017). 132 https://doi.org/10.16157/j.issn.0258-7998.2017.04. 032

37. Ministry of Housing and Urban-Rural Development of the People's Republic of China, in Gb50034-2013 standard, Lighting design of buildings. (China Architecture and Building Press, China, 2013), pp. 22-40

38. Ministry of Housing and Urban-Rural Development of the People's Republic of China, in Gb50096-2011 standard, Design code for residential buildings. (China Architecture and Building Press, China, 2011), pp. 9-10

39. I. W. B Institute, The well building standard. (Delos Living LLC, USA, 2014), p. 150. https://www.wellcertified.com/sites/default/files/resources/WELL \%20Building\%20Standard\%20-\%200ct\%202014.pdf. Accessed 2 June 2018

40. A Goldsmith, in Wireless communications. 1st edn. Wireless Communications (Cambridge University Press, UK, 2005), pp. 450-452

41. W Li, J Lei, T Wang, C Xiong, J Wei, Dynamic optimization for resource allocation in relay-aided ofdma systems under multiservice. IEEE Trans. Veh. Technol. 65(3), 1303-1313 (2016). https://doi.org/10.1109/TVT.2015. 2410832

\section{Submit your manuscript to a SpringerOpen ${ }^{\circ}$ journal and benefit from:}

- Convenient online submission

- Rigorous peer review

- Open access: articles freely available online

- High visibility within the field

- Retaining the copyright to your article

Submit your next manuscript at $\gg$ springeropen.com 